\title{
Avaliação da Satisfação do Cliente em Serviços de Restaurantes com Aplicação do ACSI
}

\section{Evaluation of Customer Satisfaction with Restaurant Services with ACSI Application}

\section{Evaluación de la Satisfacción del Cliente en Servicios de Restaurantes con Aplicación del ACSI}

\author{
Derli Luís Angnes ${ }^{1}$ \\ Carlos Alberto Mello Moyano² \\ Jorge Francisco Bertinetti Lengler ${ }^{3}$
}

\begin{abstract}
Resumo: O Brasil possui mais de um milhão de bares e restaurantes, que correspondem aproximadamente a $40 \%$ do PIB do turismo no país. Restaurantes são organizações empresariais do ramo de serviços de refeições, que, além de proporcionarem satisfação individual e convívio social, exercem importância fundamental na saúde das pessoas. 0 objetivo principal deste estudo foi validar um modelo de satisfação do cliente para o ambiente de serviços de restaurantes, tendo como referência o modelo e a metodologia do Índice Norte-Americano de Satisfação do Cliente (ACSI), a partir de uma survey com 270 casos. A metodologia caracterizou-se por um estudo exploratório qualitativo, para compreensão dos atributos relevantes nos serviços de restaurantes e, uma avaliação descritiva, com abordagem quantitativa a partir de uma alteração do modelo ACSI. A análise dos dados envolveu estatística multivariada por modelagem de equações estruturais. Os principais resultados na etapa exploratória resultaram em uma lista de 27 atributos de julgamento para serviços de restaurantes e as análises com modelagem de equações estruturais utilizadas na validação do modelo sugerem que o relacionamento, a qualidade e o valor percebido pelo cliente influenciam a sua satisfação e a lealdade com os restaurantes avaliados.
\end{abstract}

Palavras-chave: Restaurantes. Marketing de serviços. Satisfação do consumidor. ACSI.

Abstract: Brazil has more than a million bars and restaurants, which are responsible for about $40 \%$ of the tourism GDP of the country. Restaurants are business organizations in the gastronomy and service sectors that besides providing individual satisfaction and social life are of great importance for people's health. The main objective of this study was to validate a model for the customer satisfaction related to the service attributes in restaurants. The American Customer Satisfaction Index (ACSI) was used as a model and methodology reference, based on a survey with 270 clients. The methodology employed was characterized by a qualitative exploratory study, for the understanding of the relevant attributes of restaurant services, and, a descriptive evaluation, with a qualitative approach based on the ACSI model and methodology. The data analysis involved multivariate statistics with structured equation modeling. The main results from the exploratory step resulted in a list of 27 evaluation attributes for restaurant services and the analyses with a modeling of structural equations used to validate this model suggest that the relationship, the quality

\footnotetext{
${ }^{1}$ Universidade de Santa Cruz do Sul - UNISC/ University College Dublin - UCD, Irlanda. E-mail: derli.angnes@gmail.com

${ }^{2}$ UNISC - Universidade de Santa Cruz do Sul. E-mail: imur@imur.com.uy

${ }^{3}$ Universidade de Santa Cruz do Sul - UNISC / University College Dublin - UCD, Irlanda. E-mail: jorge@lengler.org
} 
and the valued experienced by the customers influence their satisfaction and loyalty towards the evaluated restaurants.

Keywords: Restaurants. Service marketing. Satisfaction. ACSI.

Resumen: En Brasil existen más de un millón de bares y restaurantes, lo que componen cerca de $40 \%$ del PIB del turismo en el país. Restaurantes son organizaciones empresariales dedicadas al servicio de comidas, que además de generar satisfacción individual y convivencia social, ejercen una importancia fundamental sobre la salud de las personas. El objetivo principal de este estudio fue definir un modelo de satisfacción de los clientes de restaurantes, tomando como referencia el modelo y la metodología del Índice Norteamericano de Satisfacción del Cliente (ACSI, en inglés), a partir de una survey con 270 casos. El método se caracterizó por un estudio exploratorio cualitativo, para la comprensión de los atributos relevantes en los servicios de restaurantes, y una evaluación descriptiva, con abordaje cuantitativa a partir de una adaptación del modelo norteamericano ACSI. El análisis de los datos fue realizado con estadística multivariada, utilizando ecuaciones estructurales. En la etapa exploratoria se identificaron 27 atributos de evaluación de los servicios de los restaurantes y los análisis con ecuaciones estructurales, utilizados en la validación del modelo, sugieren que las relaciones, la calidad y el valor percibido por el cliente influyen en su satisfacción y lealtad.

Palabras clave: Restaurantes. Marketing de servicios. Satisfacción del consumidor. ACSI.

\section{INTRODUÇÃO}

A satisfação do cliente exerce uma importante função na decisão de consumo, sua compreensão e mensuração podem ser consideradas como fatores críticos de sucesso para organizações de bens de consumo e serviços, tendo em vista que maiores índices de satisfação do cliente tendem a uma maior recompra (Lam, Shankar, Erramilli, \& Murthy, 2004).

Os primeiros estudos relacionados com a satisfação do consumidor são da década de 1960 e foram realizados por Cardozo (1965). Já uma das primeiras tentativas na mensuração da satisfação foi proposta por Oliver (1980), que definiu a satisfação do cliente como uma função da diferença entre expectativa do cliente e o desempenho do serviço/produto. A partir de então, inúmeros estudos e trabalhos foram desenvolvidos e publicados acerca do tema.

Entre as diversas metodologias existentes para medição da satisfação do consumidor, encontra-se o modelo norte-americano de avaliação da satisfação do cliente, denominado de American Customer Satisfaction Index (ACSI), proposto por Fornell, Jonhson, Anderson, Cha e Bryant (1996) para a obtenção de um indicador de satisfação de quão favorável é a avaliação dos consumidores quanto ao pós-consumo para produtos e serviços.

Muitos países, incluindo o Brasil, passaram a replicar e adaptar o modelo e a metodologia ACSI para mensurar a satisfação dos clientes de empresas e setores de sua economia, a exemplo do Índice da Agência Nacional de Energia Elétrica (ANEEL) de Satisfação do Consumidor (IASC) proposta por Marchetti e Prado (2004). Apesar de outros estudos terem sido realizados no país envolvendo a metodologia ACSI (Urdan \& Rodrigues, 1999; Moura \& Gonçalves, 2005; Lopes, Pereira, \& Vieira, 2009), nenhum trabalho consistente foi identificado envolvendo um modelo de satisfação para o setor de serviços em restaurantes no Brasil.

O objetivo principal deste artigo consiste em validar um modelo de satisfação do cliente para o ambiente de serviços de restaurantes, tendo como referência o modelo e a metodologia 
ACSI. Dessa forma, busca-se contribuir para a compreensão das relações de causa e efeito dos antecedentes e consequentes da satisfação do cliente em serviços de restaurantes, setor que exerce importância social e relevância na economia brasileira, sobretudo para a área do turismo. Para tanto, realizou-se uma alteração do modelo ACSI original, substituindo-se o construto antecedente: expectativa, pelo construto relacionamento, identificado como sendo um construto relevante, segundo a pesquisa exploratória qualitativa conduzida para serviços dessa natureza e, também, segundo referências secundárias. A pesquisa empírica envolvendo uma amostra de 72 clientes de restaurantes na etapa exploratória qualitativa e 270 casos na etapa descritiva, a partir de um levantamento (survey) com clientes de restaurantes em Santa Cruz do Sul do estado do Rio Grande do Sul.

\section{REFERENCIAL TEÓRICO}

A primeira tentativa de criar um índice nacional de satisfação de clientes ocorreu na Suécia, em 1989, e se chamou Swedish Customer Satisfaction Barometer (SCSB). Em 1992, a Alemanha também desenvolve o seu índice de satisfação do cliente, chamado de Deutsche Kundenbarometer (DK) (Fornell et al., 1996). Nos Estados Unidos, a necessidade de mensurar a satisfação dos clientes de diversos setores da economia norte-americana envolveu os esforços conjuntos das instituições American Society for Quality Control e da Faculdade de Administração da University of Michigan, que, juntas criaram o Índice Norte-Americano de Satisfação do Cliente (American Customer Satisfaction Index), em 1994 (Bateson \& Hoffman, 2001).

A validação do modelo e sua aceitação entre os especialistas tornaram o ACSI uma referência para mensuração da satisfação do cliente. Em 1998, a partir do modelo norteamericano, foi lançado o projeto-piloto do Índice Europeu de Satisfação do Cliente (ECSI), com o propósito de adequar o modelo ACSI ao mercado europeu (Lopes, Pereira, \& Vieira, 2009). 0 modelo estrutural ACSI e suas relações entre as variáveis é apresentado na Figura 1.

Figura 1 - O Modelo ACSI com suas variáveis, relações e suas hipóteses

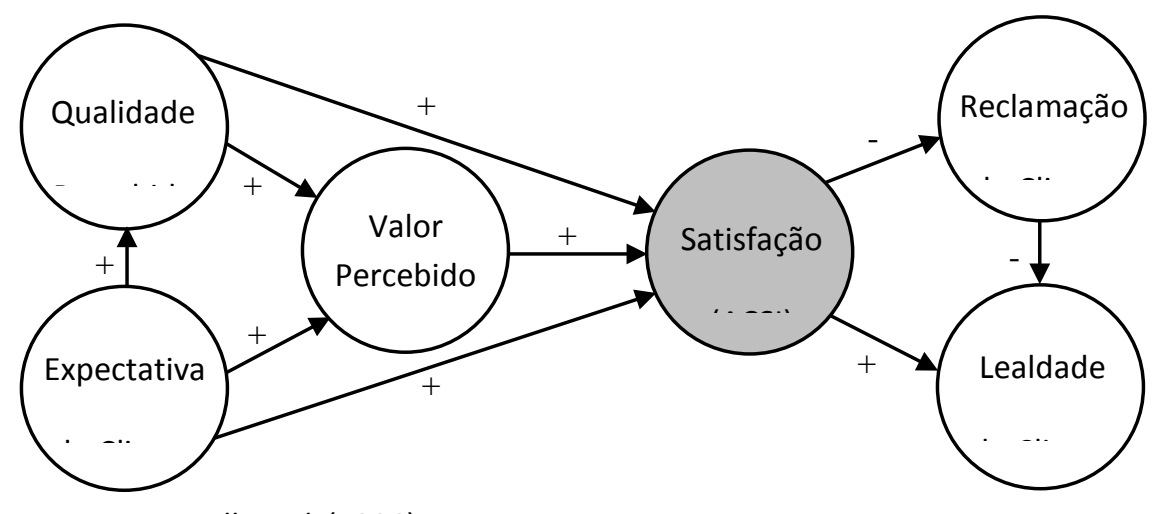

Fonte: Fornell et al. (1996) 
O modelo ACSI apresenta fundamentalmente duas propriedades: a primeira delas é que ele deve ser o suficientemente genérico para ser utilizado por diversas organizações e países; a segunda é que o modelo seja capaz de estabelecer um sistema de relações de causa e efeito que vão desde antecedentes da satisfação até os consequentes da satisfação do consumidor.

O modelo sustenta que os construtos: Expectativa do cliente, qualidade percebida e valor percebido antecedem a satisfação do cliente. O construto central do modelo ACSI é a satisfação global do cliente, mensurada pela teoria da desconfirmação da expectativa por meio de três indicadores: (1) uma medição da satisfação geral; (2) uma medição do desempenho relativo à expectativa; e (3) uma medição de comparação com o ideal. O Índice ACSI é calculado com base em uma fórmula específica e considera os três indicadores do construto da satisfação global. $O$ resultado é um escore com uma pontuação de 0 a 100 que permite comparações entre empresas do mesmo ramo e setores da economia.

Com relação às variáveis consequentes, o modelo sustenta que um aumento do índice de satisfação do cliente reduz as reclamações e eleva a lealdade do cliente. Uma síntese dos principais conceitos das variáveis do modelo e seus autores estão apresentados na Tabela 1.

Tabela 1- Síntese dos Principais Conceitos do Modelo ACSI

\begin{tabular}{|c|c|c|c|}
\hline Classificação & Construto & Definição & Autores \\
\hline \multirow{3}{*}{ Antecedentes } & Expectativa & $\begin{array}{l}\text { Reflete os desejos dos consumidores e } \\
\text { permite comparar aquilo que o cliente } \\
\text { esperava receber de serviço e aquilo que } \\
\text { ele efetivamente recebeu da empresa. }\end{array}$ & $\begin{array}{l}\text { Oliver (1980), Spreng e Mackenzie, } \\
\text { Olshavsky (1996), } \\
\text { Fornell et al. (1996), Zeithaml (1988). }\end{array}$ \\
\hline & $\begin{array}{l}\text { Qualidade } \\
\text { Percebida }\end{array}$ & $\begin{array}{l}\text { É a avaliação que o cliente faz do bem } \\
\text { físico ou serviço com base em dois } \\
\text { fatores: customização e confiabilidade. }\end{array}$ & $\begin{array}{l}\text { Fornell et al. (1996), Zeithaml, Berry } \\
\text { e Parasuraman (1996); } \\
\text { Zeithaml e Bitner (2003). }\end{array}$ \\
\hline & $\begin{array}{l}\text { Valor } \\
\text { Percebido }\end{array}$ & $\begin{array}{l}\text { É a qualidade percebida pelo mercado de } \\
\text { um bem físico ou serviço, ajustada pelo } \\
\text { seu preço relativo. }\end{array}$ & $\begin{array}{l}\text { Woodruff (1997), Parasuraman e } \\
\text { Grewal (2000). }\end{array}$ \\
\hline $\begin{array}{l}\text { Variável } \\
\text { central }\end{array}$ & Satisfação & $\begin{array}{l}\text { É a avaliação realizada pelo cliente de um } \\
\text { bem físico ou serviço, para satisfazer as } \\
\text { suas necessidades e expectativas. }\end{array}$ & $\begin{array}{l}\text { Oliver (1980), Churchill e Suprenant } \\
\text { (1982), Zeithaml e Bitner (2003). }\end{array}$ \\
\hline \multirow[b]{2}{*}{ Consequentes } & Reclamação & $\begin{array}{l}\text { É o resultado da insatisfação do cliente } \\
\text { com relação a um bem físico ou serviço. }\end{array}$ & $\begin{array}{l}\text { Zeithaml e Bitner (2003), } \\
\text { Mowen e Minor (2003). }\end{array}$ \\
\hline & Lealdade & $\begin{array}{l}\text { É a consequência de várias interações } \\
\text { entre as partes, nas quais o consumidor } \\
\text { adquire confiança nos serviços da } \\
\text { empresa. }\end{array}$ & $\begin{array}{l}\text { Fornell (1992), Anderson, Fornell e } \\
\text { Lehmann (1994), Andreassen e } \\
\text { Lindestad (1998). }\end{array}$ \\
\hline
\end{tabular}

Fonte: Adaptado de Lopes, Pereira e Vieria (2009)

O Índice ACSI é calculado com base em uma fórmula específica e considera os três indicadores do construto da satisfação global (satisfação geral, desconfirmação da expectativa e comparação com o ideal). 0 resultado é um escore com uma pontuação de 0 a 100 que permite comparações entre empresas do mesmo ramo e setores da economia. Ao longo de uma série 
histórica anual envolvendo resultados de Índices ACSI, Fornell (2007) observou que as empresas do setor de bens de consumo ou manufatura apresentam um desempenho superior, se comparado com empresas do setor de serviços. Uma possível hipótese para o desempenho inferior do setor de serviços com a satisfação dos consumidores poderia estar relacionada a maior complexidade em se avaliar serviços, se comparado aos bens de consumo.

Diversos trabalhos e publicações surgiram no Brasil a partir do Modelo ACSI. A exemplo da proposta de Marchetti e Prado (2004) à Agência Nacional de Energia Elétrica (ANEEL), que partindo do modelo e da metodologia elaborada por Fornell et al. (1996), desenvolveram o Índice ANEEL de Satisfação do Consumidor (IASC), para monitorar o desempenho das concessionárias de energia elétrica no país a partir de indicadores globais e específicos de mensuração da satisfação dos consumidores de energia elétrica no país.

A partir da difusão do ACSI em estudos de diversas áreas e setores no Brasil é que surgiu o BCSI - Índice Brasileiro de Satisfação de Clientes, cujo seu objetivo é proporcionar indicadores para avaliação da competitividade de empresas brasileiras que aderirem ao modelo, permitido inclusive comparações de desempenho em nível global, tendo em vista que o modelo é utilizado por 17 países com mais de 40 setores estudados.

\subsection{Modelo e Desenvolvimento de Hipóteses}

Na pesquisa empírica deste trabalho, substituiu-se o constructo da expectativa do cliente por relacionamento. A substituição é justificada pelos próprios autores Fornell et al. (1996), que consideraram a expectativa uma medida imperfeita no modelo, pois deveria ser medida antes do consumo (pré-compra) e não no pós-consumo (pós-encontro) como ocorre com as demais variáveis do modelo. A inclusão do constructo relacionamento se justifica pelo fato dos mesmos autores considerarem o relacionamento um antecedente influenciador no modelo, além disso, houve uma forte representatividade dada ao atributo relacionamento no atendimento em restaurantes, segundo amostra de clientes consultados na pesquisa exploratória deste estudo. Para Zineldin (2006) o relacionamento exerce importância na geração de valor superior e na satisfação do cliente.

Na sequência, são descritas as hipóteses do modelo adaptado para o setor de restaurantes, tendo como base o modelo desenvolvido por Fornell et al (1996) e demais considerações supracitadas.

Hipótese H1: o relacionamento com o cliente é positivamente relacionado com a qualidade percebida do cliente (Santos \& Fernandes, 2008; Mello, 2010).

Hipótese H2: a qualidade percebida é positivamente relacionada com o valor percebido pelo cliente (Fornell et al., 1996).

Hipótese H3: o relacionamento com o cliente é positivamente relacionado com o valor percebido pelo cliente (Zineldin, 2006). 
Hipótese H4: a qualidade percebida é positivamente relacionada com a satisfação do cliente (Fornell et al., 1996).

Hipótese H5: o valor percebido pelo cliente é positivamente relacionado com a satisfação do cliente (Fornell et al., 1996).

Hipótese H6: o relacionamento com o cliente é positivamente relacionado com a satisfação do cliente (Zineldin, 2006).

Hipótese H7: a satisfação do cliente é negativamente relacionada com a reclamação do cliente (Fornell et al., 1996).

Hipótese H8: a satisfação do cliente é positivamente relacionada com a lealdade do cliente (Fornell et al., 1996).

Hipótese H9: a reclamação do cliente é relacionada negativamente com a lealdade do cliente (Fornell et al., 1996).

A Figura 2 apresenta o Modelo Hipotético da satisfação para os serviços de restaurantes.

Figura 2- O Modelo Hipotético com suas variáveis, relações e suas hipóteses

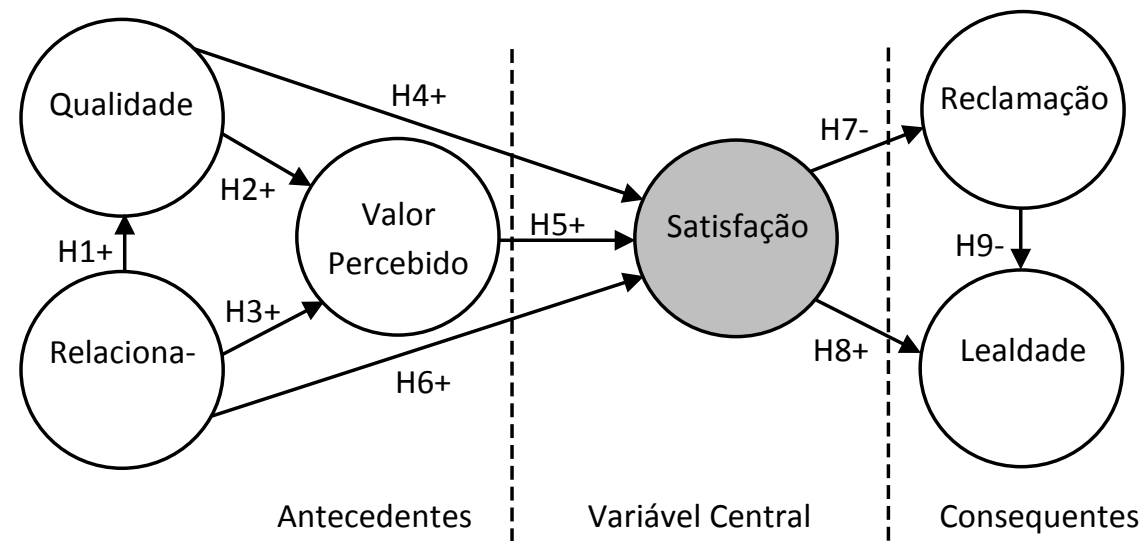

Fonte: Fornell et al. (1996)

\section{METODOLOGIA DA PESQUISA}

A pesquisa empírica deste estudo é de natureza aplicada e de caráter exploratória e descritiva transversal única (Malhotra, 2012). Com abordagem de análise qualitativa no estágio exploratório e com análise quantitativa no estágio descritivo. No estágio exploratório a coleta dos dados ocorreu mediante aplicação da técnica do incidente crítico e análise de conteúdo, cujos resultados foram publicados por Angnes e Mello Moyano (2013). No estágio descritivo a coleta dos dados primários se deu por levantamento (Survey) e a análise dos dados foi realizada por meio de estatística descritiva univariada e multivariada. Na sequência, estão apresentadas as etapas que definem melhor a metodologia empregada no estudo. Neste estudo daremos maior ênfase aos resultados quantitativos da pesquisa.

Primeira etapa: constituiu-se de um estudo exploratório qualitativo para identificar os atributos relevantes nos serviços de restaurantes. Adotou-se como procedimento de pesquisa a 
utilização de entrevistas com questionário não estruturado a partir da técnica do incidente crítico. Para Malhotra (2012), a pesquisa exploratória auxilia na compreensão do problema de pesquisa e na obtenção de dados adicionais, caracterizando-se por um processo de pesquisa flexível e não estruturado. Dentre as técnicas disponíveis para estudos exploratórios, optou-se pela técnica do incidente crítico, proposta e difundida inicialmente por Flanagan (1954) e empregada como técnica metodológica para estudos de pesquisa em marketing (Lockwood, 1994). A técnica do incidente crítico consiste no levantamento de relatos de experiências satisfatórias (positivas) e insatisfatórias (negativas) com clientes.

Para obtenção dos dados, utilizou-se uma entrevista por conveniência com 72 clientes de restaurantes em Santa Cruz do Sul no Rio Grande do Sul, número acima da média recomenda para estudos dessa natureza, tendo como critério de público-alvo: homens e mulheres acima de 15 anos que tivessem almoçado em algum restaurante, em um período inferior a 30 dias. Para coleta dos incidentes, utilizou-se um questionário não estruturado. Cada um dos entrevistados descreveu até cinco experiências de incidentes positivos e cinco experiências negativas que vivenciou ao ter frequentado o último restaurante. A análise de conteúdo foi realizada por dois especialistas em marketing. A técnica se constituiu em analisar incidentes (positivos e negativos) e agrupá-los pelo critério de repetição ou semelhança no significado das palavras (análise de conteúdo). Para cada grupo de incidentes (palavras) semelhantes, nomeou-se um atributo. A coleta e análise dos dados ocorreram em 2012 (Angnes \& Mello Moyano, 2013).

Segunda etapa: posteriormente à fase exploratória qualitativa, deu-se continuidade com a fase descritiva. Para Malhotra (2012), o estudo descritivo é recomendado para situações onde se deseja testar hipóteses e responder a questões específicas da pesquisa.

O procedimento adotado nesta etapa da pesquisa foi o Levantamento, ou Survey, por meio de questionário estruturado e com escala de diferencial semântico de sete pontos. O questionário foi elaborado tendo como referência os indicadores do questionário empregado pela Metodologia ACSI proposta por Fornell et al. (1996) e adaptado para os serviços de restaurantes, com a incorporação dos atributos identificados na fase exploratória. O questionário foi aplicado para uma amostra não probabilística por quotas de sexo e faixa etária, com 270 clientes que almoçaram num período de 30 dias em algum restaurante de Santa Cruz do Sul no Rio Grande do Sul. Ainda, segundo Hair, Anderson, Tatham, Black e Babin (2009), considerando-se 18 questões (três indicadores por construto), 51 parâmetros a serem estimados e ainda cinco respondentes por parâmetro, ter-se-ia como amostra mínima 255 casos. Optou-se por elevar esse número para 270 casos. A Tabela 2 relaciona as variáreis (indicadores) utilizadas na medição dos construtos. 
Tabela 2- Variáveis de Medição Usadas no Questionário

\begin{tabular}{|c|c|c|}
\hline Questão & Variável de medição & Construto \\
\hline Q01 & $\begin{array}{l}\text { Avaliação da experiência de confiabilidade dos serviços por meio da ausência de } \\
\text { problemas na infraestrutura do restaurante. }\end{array}$ & \multirow{3}{*}{$\begin{array}{l}\text { Qualidade } \\
\text { percebida }\end{array}$} \\
\hline Q02 & $\begin{array}{l}\text { Avaliação da experiência de personalização dos serviços nas exigências pessoais do } \\
\text { cliente com o restaurante avaliado. }\end{array}$ & \\
\hline Q03 & Avaliação global da experiência de qualidade no serviço de restaurante. & \\
\hline V04 & Classificação da qualidade em infraestrutura relativa ao preço praticado. & \multirow{3}{*}{ Valor percebido } \\
\hline V05 & Classificação da qualidade em personalização do serviço relativa ao preço praticado. & \\
\hline V06 & Avaliação global dos benefícios obtidos relativo ao preço pago. & \\
\hline RL07 & Avaliação do atendimento dos garçons e funcionários do restaurante. & \multirow{3}{*}{ Relaciona-mento } \\
\hline RL08 & Avaliação do tempo de atendimento no restaurante avaliado. & \\
\hline RL09 & Avaliação global do relacionamento no restaurante. & \\
\hline S10 & Satisfação global do restaurante avaliado. & \multirow{3}{*}{ Satisfação (ACSI) } \\
\hline S11 & Nível de expectativa do cliente em relação ao serviço do restaurante. & \\
\hline S12 & Desempenho do serviço no restaurante avaliado. & \\
\hline L13 & Tolerância de preço (diminuição) para induzir recompra. & \multirow{3}{*}{$\begin{array}{l}\text { Lealdade do } \\
\text { cliente }\end{array}$} \\
\hline L14 & Tolerância de preço (aumento) considerando recompra. & \\
\hline L15 & Avaliação global da lealdade do cliente com o restaurante avaliado. & \\
\hline R16 & Avaliação da reclamação formal ou informal do cliente no restaurante. & \multirow{3}{*}{$\begin{array}{l}\text { Reclamação de } \\
\text { cliente }\end{array}$} \\
\hline R17 & Avaliação da capacidade do restaurante em solucionar problemas. & \\
\hline R18 & Avaliação global das soluções a partir das reclamações do cliente. & \\
\hline
\end{tabular}

Fonte: Adaptado de Fornell et al. (1996).

Terceira Etapa: o processo de análise dos dados coletados iniciou com a análise univariada descritiva e, em seguida, foi realizada a análise multivariada com emprego da análise Fatorial Confirmatória para validação do modelo de medida e da análise com Equações Estruturais, para avaliação estrutural do modelo ACSI modificado para os serviços de restaurantes. A análise multivariada levou em consideração as referências de Brei e Liberali Neto (2006), Hair et al. (2009) e Malhotra (2012). A análise dos dados se deu mediante recurso computacional com a utilização dos softwares aplicativos Microsoft Excel ${ }^{\circledast}$ e SPSS/AMOS ${ }^{\circledast}$ (Statistical Package for the Social Sciences).

\section{ANÁLISE DOS RESULTADOS}

A análise dos resultados está organizada em quatro seções. A primeira seção aborda a análise exploratória dos atributos em serviços de restaurantes, uma das contribuições do artigo. Ainda nessa seção, é feita uma breve análise descritiva da amostra. Na segunda seção, aborda-se a análise e validação do modelo de medida. Na terceira seção, descreve-se a análise e validação do modelo estrutural ACSI modificado para restaurantes. Por fim, na quarta seção, apresenta-se o desempenho do índice ACSI dos restaurantes avaliados em Santa Cruz do Sul. 


\subsection{Análise exploratória e descritiva}

A entrevista foi realizada com 72 clientes de restaurantes entre 17 a 55 anos, com média de idade de 26 anos, dos quais 50\% eram homens e 50\% mulheres. Foram validados e utilizados 615 incidentes críticos para análise de conteúdo. Uma listagem final, após análise de conteúdo, resultou em 27 atributos que foram correlacionados com o composto mercadológico do marketing de serviços (Angnes \& Mello Moyano, 2013). Dentre os atributos listados e apresentados na Tabela 3, há uma forte presença de atributos de busca, caracterizados pela sua tangibilidade nos serviços (Zeithaml, 1981). A relação de atributos identificada pela pesquisa exploratória é semelhante a de outras pesquisas (Tinoco \& Ribeiro, 2008, Souki et al., 2010). Apenas destaca-se a ausência do atributo - segurança - presente em um dos estudos realizado em uma capital brasileira. Os atributos foram utilizados para adaptação do instrumento de medida ACSI proposta por Fornell et al. (1996), sem, com isso, alterar a intenção de medição das variáveis originalmente utilizadas pelo ACSI para cada um dos constructos do modelo. Para maiores informações sobre essa etapa da pesquisa, sugere-se uma consulta ao artigo publicado por Angnes e Mello Moyano (2013). Sendo que os principais resultados encontrados estão apresentados na Tabela 3. A primeira coluna apresenta a frequência de citações do atributo pelos entrevistados, na segunda coluna é apresentado o termo do atributo e na terceira coluna, o enquadramento dos atributos para cada dimensão no composto mercadológico.

Tabela 3 - Atributos em Serviço de Restaurante

\begin{tabular}{|c|c|c|c|c|c|}
\hline Frequência & Atributos & Dimensão & Frequência & Atributos & Dimensão \\
\hline $5 \%$ & Localização & \multirow{2}{*}{ Praça } & $1 \%$ & Funcionários & \multirow{5}{*}{ Pessoas } \\
\hline $6 \%$ & Estacionamento & & $2 \%$ & Garçons & \\
\hline $2 \%$ & Espaço físico & \multirow{9}{*}{$\begin{array}{l}\text { Ambiente } \\
\text { Physical } \\
\text { Environment }\end{array}$} & $1 \%$ & Simpatia & \\
\hline $2 \%$ & Infraestrutura interna & & $1 \%$ & Uniforme & \\
\hline & miraestrucura incerna & & $11 \%$ & Atendimento & \\
\hline $3 \%$ & Climatização & & $2 \%$ & Qualidade & \multirow{7}{*}{ Processo } \\
\hline $1 \%$ & Iluminação & & $2 \%$ & Organização & \\
\hline $4 \%$ & Higiene & & & Politica nara fumantes & \\
\hline $3 \%$ & Limpeza & & $1 \%$ & Politica para fumantes & \\
\hline $2 \%$ & Banheiros & & & Horárin de atendimento & \\
\hline $8 \%$ & Ambiente & & $3 \%$ & Horarıo de atenaimento & \\
\hline $13 \%$ & Comidas e pratos & \multirow{5}{*}{ Produto } & $1 \%$ & Agilidade & \\
\hline \multirow{2}{*}{$5 \%$} & \multirow{2}{*}{$\begin{array}{l}\text { Variedade no buffet } \\
\text { (cardápio) }\end{array}$} & & $9 \%$ & $\begin{array}{l}\text { Preço/Condições de } \\
\text { pagamento }\end{array}$ & Preço \\
\hline & & & $2 \%$ & Música & Promoção \\
\hline $2 \%$ & Sobremesas & & $9 \%$ & Incidentes desconsiderado & \\
\hline $2 \%$ & Bebidas & & $100 \%$ & & \\
\hline
\end{tabular}

Fonte: Angnes e Mello Moyano (2013) 
Na etapa posterior, descritiva, foram aplicados 436 questionários, dos quais apenas 270 (62\%) foram validados e aproveitados para atender aos critérios do plano amostral, sendo, 128 (47\%) compostos por homens e 142 (53\%) mulheres. A média de idade foi de 40 anos. No total, foram avaliados 44 restaurantes. As próximas seções apresentam os resultados dessa etapa da pesquisa.

\subsection{Avaliação do modelo de medida}

A análise do modelo de medida busca avaliar o quanto o modelo teórico é capaz de reproduzir a estrutura correlacional das variáveis observadas na amostra em estudo, a partir da Análise Fatorial Confirmatória sob seis perspectivas principais: (1) diagrama de caminhos; (2) exame da consistência das estimativas dos parâmetros; (3) avaliação do modelo por meio de índices de ajuste; (4) análise dos resíduos; e (5) análise dos índices de modificação e (6) validade e confiabilidade do modelo. Cada uma das perspectivas será analisada na sequência com base em Brei e Liberali Neto (2006), Hair et al. (2009) e Malhotra (2012). Para estimação do modelo, foi adotado o método de máxima verossimilhança (ML).

- (1) Diagrama de caminhos: as estimativas padronizadas do modelo com os seus fatores apresentaram carga fatorial superior a 0,5, com exceção do indicador R16, que tinha a sua escala invertida e foi removida do modelo.

- (2) Estimativas dos parâmetros: todas as estimativas dos parâmetros do modelo foram bastante significativas estatisticamente (valor $p<0,001$ ). Os erros padrão (S.E) ficaram próximos a zero e a razão crítica (C.R) foi superior a 1,96 em todas as avaliações. O que evidencia um bom ajuste e qualidade do modelo, além da robustez teórica.

- (3) Índice de ajuste: todos os índices selecionados e apresentados pela Tabela 4, referente ao modelo, se mantiveram no geral com um bom ajuste.

Tabela 4- Principais Índices de Ajuste do Modelo

\begin{tabular}{lllll}
\hline Medida & Índice & Resultado & Referência & Consideração \\
\hline & NPAR & 50 & ---- & $N^{\circ}$ de Parâmetros \\
& DF & 103 & --- & Graus de Liberdade \\
Índices absolutos & CMIN/DF & 1,324 & $\leq 5$ & Ótimo \\
& RMR & 0,042 & Mais próximo de 0 & Adequado \\
& GFI & 0,944 & $\geq 0,9$ & Bom \\
& AGFI & 0,917 & $\geq 0,9$ & Bom \\
Índices relativos & NFI & 0,943 & & \\
& RFI & 0,925 & & Bom \\
& IFI & 0,985 & $>0,90$ & \\
\hline Índices de discrepância & RMSEA & 0,035 & $>0,05$ & Um pouco abaixo \\
\hline
\end{tabular}

Fonte: Elaborado pelos autores 
- (4) Análise de resíduos: apenas um caso de resíduo foi identificado com um pouco de elevação. Mesmo assim, optou-se por manter a variável em relação ao bom desempenho geral da avaliação no todo.

- (5) Análise dos índices de modificação: embora a análise houvesse indicado duas melhorias no ajuste do modelo, sua inclusão não foi utilizada por ausência de consistência teórica e pela pouca diferença do índice de modificação em relação aos demais resultados.

- (6) Validade e confiabilidade do modelo: De acordo com Akyol e Akehurst (2003), a validação de conteúdo de uma escala é mais qualitativa do que quantitativa. A validade de conteúdo foi avaliada através de uma revisão de literatura e consultando-se pesquisadores experientes na área. Com base nas contribuições dos especialistas, conclui-se que as medições utilizadas neste estudo são válidas. Todas as cargas fatoriais das variáveis padronizadas foram acima de 0,54 , com exceção de um indicador do construto lealdade que ficou com 0,45 . 0 alfa de Cronbach variou de 0,580 (lealdade) a 0,861 (relacionamento). Os resultados para a confiabilidade composta variaram de 0,58 (lealdade) a 0,87 (relacionamento). Segundo Malhotra (2012) estimativas entre 0,6 e 0,7 podem ser aceitáveis se as estimativas da validade do modelo forem boas. Embora o construto lealdade tenha apresentado 0,58, optou-se por mantê-lo em razão do bom desempenho geral do modelo.

A partir das avaliações supracitadas por meio da Análise Fatorial Confirmatória, constatouse que o modelo de medida descreve adequadamente e com cientificidade os dados amostrais da pesquisa empírica.

\subsection{Avaliação do modelo estrutural}

Após a validação do modelo de medida, mediante a Análise Fatorial Confirmatória, inicia-se o processo de avaliação do modelo estrutural para verificar a relação entre os fatores (construtos) e testar suas hipóteses. A Figura 3 apresenta o diagrama visual do modelo estrutural.

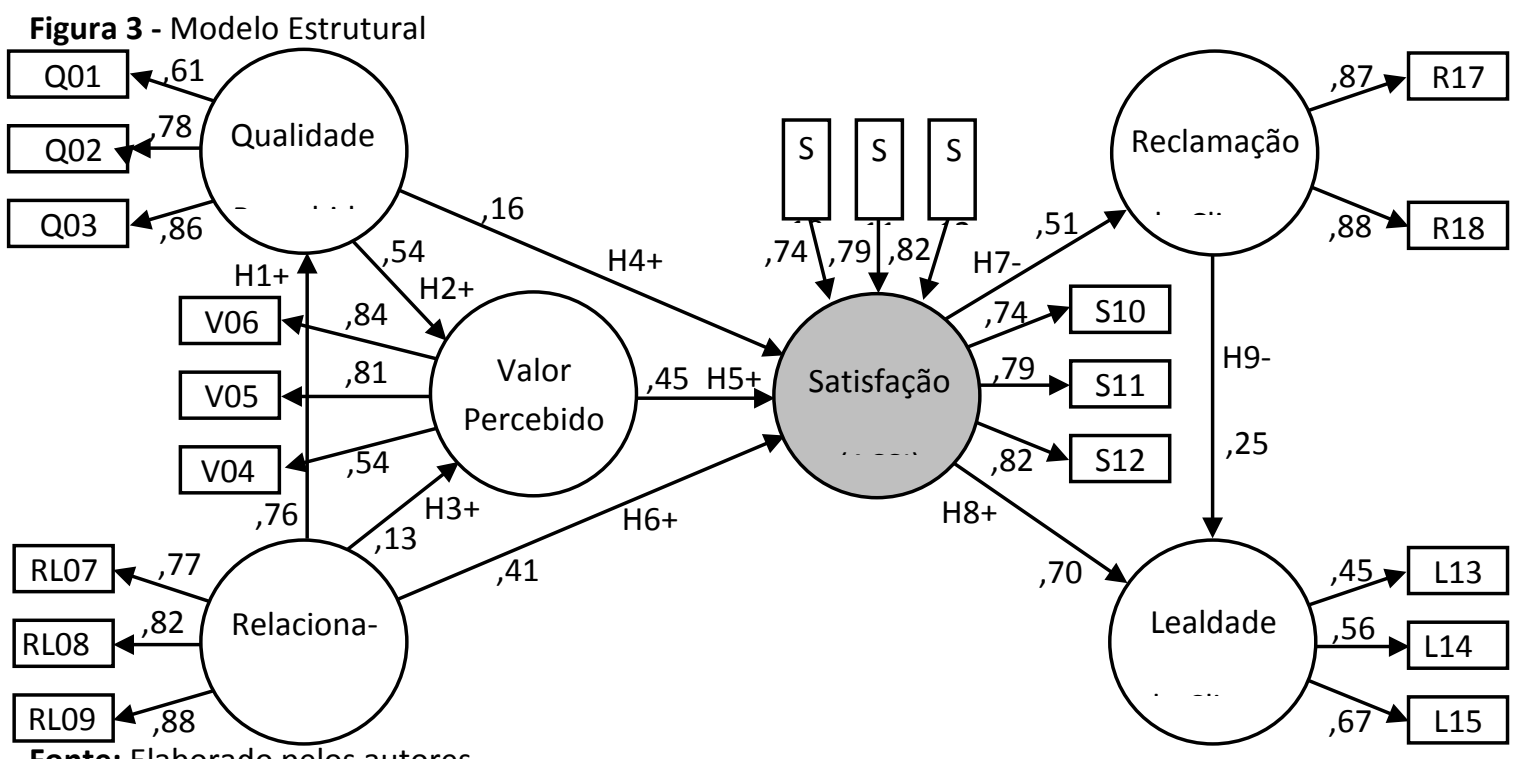

Fonte: Elaborado pelos autores 
As linhas representam os caminhos e suas relações. Os retângulos são os indicadores de medição e os construtos estão representados pelos círculos. Os valores ao lado das setas indicam as estimativas paramétricas estruturais resultantes completamente padronizadas.

O vínculo entre relacionamento e qualidade percebida foi o mais expressivo na análise do modelo estrutural, com um coeficiente de 0,76 , o que representa que quanto maior for o relacionamento dos restaurantes com o cliente, maior será a qualidade percebida. Outro vínculo forte e expressivo da análise foi entre os pares da satisfação e lealdade, com um coeficiente de 0,70 . O que evidencia que, quanto maior a satisfação com os serviços de restaurantes, maior será a lealdade dos clientes. A Tabela 5 apresenta os resultados verificados nos testes das hipóteses estabelecidos no modelo estrutural.

Tabela 5 - Teste das Hipóteses do Modelo

\begin{tabular}{|c|c|c|c|c|c|c|c|c|}
\hline HIPÓTESE & DESCRIÇ & Ão D & A HIPÓTESE & $\begin{array}{c}\text { Estimativa } \\
\text { Padronizada }\end{array}$ & Estimativa & S.E. & $\mathbf{p}$ & $\begin{array}{c}\text { Resultado da } \\
\text { Hipótese }\end{array}$ \\
\hline $\mathrm{H} 1+$ & Qualidade & $\leftarrow$ & Relacionamento & 0,76 & 0,622 & 0,072 & $*$ & ACEITA \\
\hline $\mathrm{H} 2+$ & Valor & $\leftarrow$ & Qualidade & 0,54 & 0,569 & 0,139 & $*$ & ACEITA \\
\hline $\mathrm{H} 3+$ & Valor & $\leftarrow$ & Relacionamento & 0,129 & 0,111 & 0,097 & 0,251 & REJEITADA \\
\hline $\mathrm{H} 4+$ & Satisfação & $\leftarrow$ & Qualidade & 0,162 & 0,171 & 0,1 & 0,085 & REJEITADA \\
\hline H5+ & Satisfação & $\leftarrow$ & Valor & 0,45 & 0,452 & 0,083 & $*$ & ACEITA \\
\hline $\mathrm{H} 6+$ & Satisfação & $\leftarrow$ & Relacionamento & 0,411 & 0,356 & 0,074 & $*$ & ACEITA \\
\hline H7- & Reclamações & $\leftarrow$ & Satisfação & 0,51 & 0,938 & 0,134 & $*$ & REJEITADA \\
\hline H9- & Lealdade & $\leftarrow$ & Reclamações & 0,25 & 0,107 & 0,037 & 0,004 & REJEITADA \\
\hline $\mathrm{H} 8+$ & Lealdade & $\leftarrow$ & Satisfação & 0,695 & 0,546 & 0,098 & * & ACEITA \\
\hline
\end{tabular}

Fonte: Elaborado pelos autores

$* p<0,01$.

Algumas hipóteses foram rejeitadas $(\mathrm{H3}, \mathrm{H} 4, \mathrm{H7}, \mathrm{H9})$, principalmente as que apresentavam hipóteses negativas e relacionadas ao construto reclamação (H7 e H9). Um modelo alternativo é então sugerido, tendo como referência a exclusão das relações, hipóteses e construtos rejeitados, conforme apresentado na Figura 4. 
Figura 4 - Modelo Alternativo

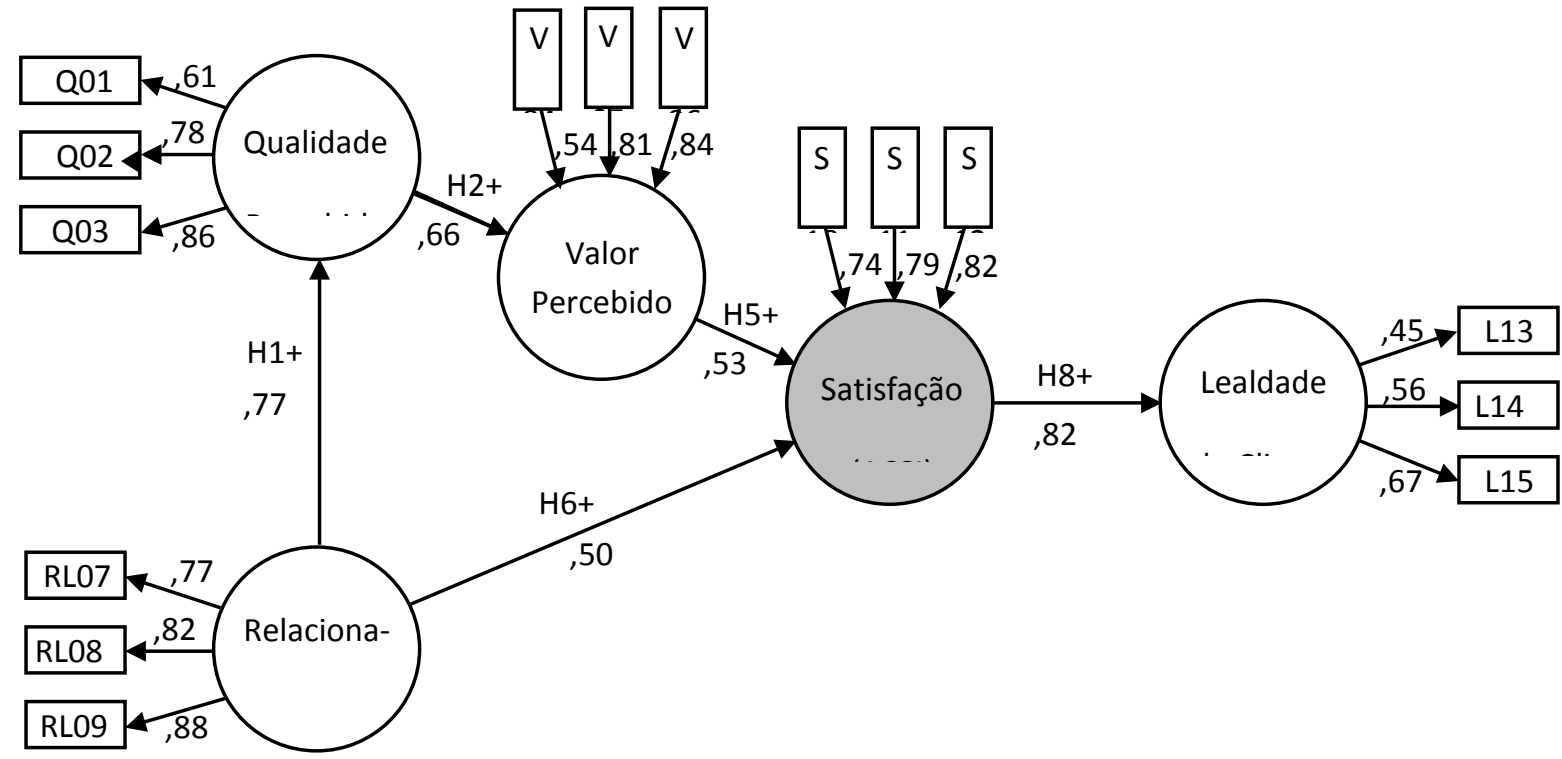

Fonte: Elaborado pelos autores

Com a alteração do modelo, todas as hipóteses foram bastante significativas e, portanto, aceitas, conforme os resultados obtidos e apresentados na Tabela 6.

Tabela 6 - Teste das Hipóteses do Modelo Alternativo

\begin{tabular}{ccccccccc}
\hline HIPÓTESE & \multicolumn{2}{c}{ DESCRIÇÃO DA HIPÓTESE } & $\begin{array}{c}\text { Estimativa } \\
\text { Padronizada }\end{array}$ & Estimativa & S.E. & P & $\begin{array}{c}\text { Resultado da } \\
\text { Hipótese }\end{array}$ \\
\hline H1+ & Qualidade & $\leftarrow$ & Relacionamento & 0,774 & 0,63 & 0,072 & $*$ & ACEITA \\
H2+ & Valor & $\leftarrow$ & Qualidade & 0,661 & 0,704 & 0,109 & $*$ & ACEITA \\
H5+ & Satisfação & $\leftarrow$ & Valor & 0,532 & 0,53 & 0,082 & $*$ & ACEITA \\
H6+ & Satisfação & $\leftarrow$ & Relacionamento & 0,495 & 0,427 & 0,057 & $*$ & ACEITA \\
H8+ & Lealdade & $\leftarrow$ & Satisfação & 0,823 & 0,619 & 0,105 & $*$ & ACEITA \\
\hline
\end{tabular}

Fonte: Elaborado pelos autores

$* p<0,01$.

Em razão da alteração do modelo, uma nova avaliação da qualidade de ajuste do modelo foi necessária, a qual se encontra apresentada na Tabela 7. 
Tabela 7 - Principais Índices de Ajuste do Modelo Alternativo

\begin{tabular}{lllll}
\hline Medida & Índice & Resultado & Referência & Consideração \\
\hline & NPAR & 35 & ---- & $N^{\circ}$ de Parâmetros \\
Índices absolutos & DF & 85 & --- & Graus de Liberdade \\
& CMIN/DF & 1,921 & $\leq 5$ & Ótimo \\
& RMR & 0,051 & Mais próximo de 0 & Adequado \\
& GFI & 0,926 & $\geq 0,9$ & Bom \\
& AGFI & 0,896 & $\geq 0,9$ & Razoável \\
Índices relativos & NFI & 0,921 & & Bom \\
& RFI & 0,902 & & \\
& IFI & 0,960 & $>0,90$ & Bom \\
& TLI & 0,950 & & Bom \\
\hline Índices de parcimônia & CFI & 0,960 & & \\
\hline Índices de discrepância & PRATIO & 0,810 & & \\
\hline
\end{tabular}

Fonte: Elaborado pelos autores

Todos os índices selecionados e apresentados pela Tabela 7, referentes ao modelo alternativo, se mantiveram, no geral, com um bom ajuste. Portanto, o modelo inicial proposto para o estudo não se confirmou, o que culminou em uma proposta alternativa com base na modificação do modelo inicial. Conclui-se, a partir da análise multivariada do modelo de medida e estrutural, que houve um ajustamento de boa qualidade, garantindo, dessa forma, a cientificidade estatística do modelo alternativo proposto. Para uma generalização do modelo, Hair et al. (2009) sugerem a realização de uma nova amostra independente daquela onde o modelo foi ajustado para conclusões definitivas.

\subsection{Avaliação do índice ACSI dos restaurantes}

A utilização de um índice de satisfação padronizado permite a comparação de desempenho entre os restaurantes e, ainda, entre o setor de restaurantes de determinado país em relação a outros restaurantes de nacionalidade diferente com base na mesma metodologia.

A Fórmula 1 apresenta a equação do Índice ACSI empregado para o cálculo do índice da Satisfação Global dos restaurantes avaliados. 
Fórmula 1 - Fórmula do Cálculo do Índice da Satisfação ACSI

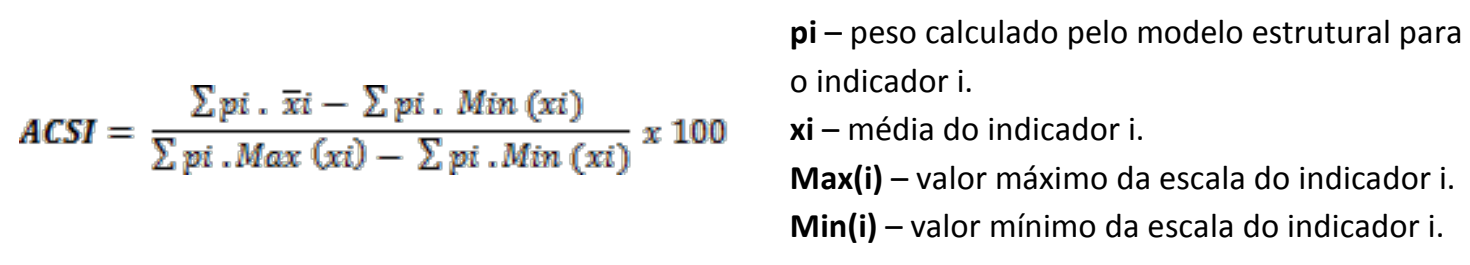

Fonte: Marchetti e Prado (2004)

A partir da obtenção do Índice de Satisfação Global dos restaurantes, é possível realizar benchmarking com outros restaurantes ou com o resultado do setor. Dados históricos do ACSI (2012) informam que o setor de restaurantes nos Estados Unidos obteve, em 2012, um índice de desempenho na satisfação de 80 pontos. Conforme dados apresentados na Tabela 8.

Tabela 8 - Comparativo de Índices de Satisfação do Setor de Alimentação

\begin{tabular}{lcccc}
\hline \multicolumn{1}{c}{ SERVIÇOS DE ALIMENTAÇÃo } & \multicolumn{3}{c}{ Índice ACSI } \\
\cline { 2 - 4 } & $\mathbf{1 9 9 4}$ & $\mathbf{2 0 0 7}$ & $\mathbf{2 0 1 2}$ \\
\hline Fast Food EUA & 69 & 77 & 73 \\
McDonald's EUA & 63 & 64 & 80 \\
Restaurantes EUA & -- & 81 & 74 \\
Restaurante avaliado em Santa Cruz do Sul (RS) Brasil & -- & -- & \\
\hline
\end{tabular}

Fonte: Adaptado de ACSI (2012)

Para interpretação do desempenho, sugere-se o critério utilizado pela Agência Nacional de Energia Elétrica (ANEEL) (2012), que utiliza a mesma metodologia para avaliar a satisfação dos consumidores de energia elétrica no Brasil. São utilizados pela ANEEL cinco critério de interpretação: para a faixa de 0 a 20 pontos, o desempenho é considerado péssimo. De 21 a 40 pontos, o desempenho é ruim. De 41 a 60 pontos, o desempenho é regular. De 61 a 80 pontos, o desempenho é bom e, para faixa de 81 a 100 pontos, o desempenho é considerado ótimo.

Os restaurantes avaliados no Brasil obtiveram um Índice ACSI de 74 pontos. O resultado sugere que o desempenho dos serviços foi satisfatoriamente bom, com possibilidades de melhoria. Segundo Ladhari (2009), o investimento na qualidade dos serviços conduz à retenção e atração de clientes, reduz os custos futuros, fortalece a marca, a imagem da empresa e eleva os ganhos financeiros.

A satisfação do cliente afeta o comportamento futuro de compra do cliente, que, por sua vez, influencia o fluxo de caixa da empresa. Existe uma associação positiva entre a satisfação do cliente com o valor para o acionista (Anderson, Fornell, \& Mazvancheryl, 2004; Fornell et al., 2006).

Para investimentos na melhoria dos serviços dos restaurantes avaliados pelo estudo, sugerese que sejam incrementadas ações estratégicas nos antecedentes da satisfação do cliente de 
restaurantes (relacionamento, qualidade e valor percebido), validados no modelo deste estudo (Figura 5), considerando-se para isso, ações específicas de melhorias nos atributos dos serviços de restaurantes, identificados na etapa exploratória qualitativa (Tabela 3).

Figura 5 - Modelo Alternativo Conclusivo

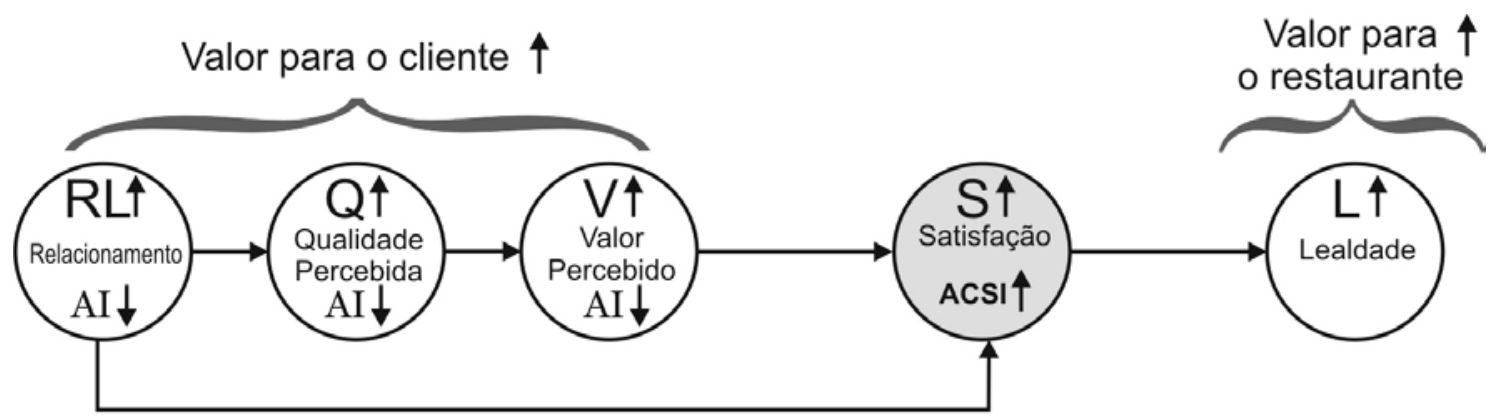

Al: Atributos de Insatisfação

Fonte: Elaborado pelos autores

A redução dos atributos de insatisfação $(\mathrm{Al})$ tenderá a promover uma elevação positiva nos indicadores das variáveis antecedentes da satisfação (S): um aumento nos níveis de relacionamento $(R L)$ com o cliente influenciará positivamente na qualidade percebida (Q) e na satisfação final (S). A melhoria da qualidade dos serviços (Q) influenciará positivamente no valor percebido pelo cliente (V) e, por conseguinte, promoverá um aumento no grau de satisfação (S) e, uma elevação na lealdade (L) com os serviços, resultado na maximização de valor em forma de receita aos empresários e acionistas do ramo de restaurantes, cujas estratégias estejam alinhadas com o modelo (Figura 4) de orientação para o marketing de restaurantes.

\section{CONSIDERAÇÕES FINAIS}

O artigo apresentado teve como objetivo validar um modelo de satisfação do cliente para o ambiente de serviços de restaurantes, tendo como referência o modelo e a metodologia ACSI, corroborando, dessa maneira, para ampliar e aprofundar os estudos da satisfação do cliente realizados no país (Urdan \& Rodrigues, 1999; Marchetti \& Prado, 2004; Moura \& Gonçalves, 2005; Lopes, Pereira \& Vieira, 2009), sobretudo para o setor de restaurantes.

As principais contribuições deste estudo podem ser consideradas de ordem acadêmica e gerencial. Sendo que a principal contribuição está amparada pelas análises com modelagem de equações estruturais que sugerem que o relacionamento, a qualidade e o valor percebido pelos clientes de restaurantes influenciam na sua satisfação e na lealdade com os serviços de restaurantes. Destacase ainda, que as hipóteses do construto relacionamento foram aceitas, evidenciando sua relevância e influência sobre a satisfação. $O$ construto reclamação não foi validado pelas análises e foi removido do modelo. Uma segunda contribuição relevante deste estudo foi a relação de 27 atributos identificados na pesquisa exploratória com os serviços de restaurantes, os quais 
proporcionam valor superior para clientes dessa natureza de serviço e representam importante subsídio para ações diretas de melhoria dos estabelecimentos (Angnes \& Mello Moyano, 2013). 0 índice ACSI de 74 pontos, obtido com base em 44 restaurantes avaliados, sugere um bom desempenho, com possibilidades de melhoria dos serviços prestados. Ações estratégicas nos antecedentes: relacionamento, qualidade e valor, a partir dos seus respectivos atributos, tendem a elevar a satisfação e promover uma maior lealdade do cliente de restaurantes.

De forma mais clara, pode-se dizer que o estudo trouxe contribuições acadêmicas ao evidenciar a validação e a importância do construto relacionamento como um antecedente da satisfação do cliente em serviços de restaurantes, tendo em vista que o modelo original não incorpora esse construto. No âmbito gerencial, o estudo contribuiu para comparar os restaurantes avaliados com a média dos restaurantes nos Estados Unidos no mesmo período coma a utilização de metodologia semelhante, além disso, o estudo trouxe contribuições também para elucidar as principais variáveis que influenciam na satisfação dos clientes de restaurantes avaliados pelo estudo. Salienta-se que embora o preço seja uma variável presente e significante nos atributos identificados na etapa qualitativa, ela não é decisiva na compra para maioria dos clientes quando pondera o benefício e a qualidade dos serviços prestados. Portanto, ações de melhorias gerenciais devem ser direcionadas no sentido de propor estratégias de diferenciação.

Salienta-se que resultados dessa pesquisa poderão ser utilizados como subsídio e referência para futuros trabalhos, envolvendo a mensuração da satisfação e do comportamento do consumidor em serviços, sobretudo para o setor de alimentação e de restaurantes no país. 0 modelo apresentado pode ser replicado em outros restaurantes na intenção de avaliar o seu comportamento em outras regiões ou cidades. Já o índice de satisfação pode ser utilizado para ranquear ou mensurar a satisfação com os serviços prestados por diferentes restaurantes avaliados sob a mesma metodologia, gerando com isso um indicador de desempenho com base científica para a área de turismo e setores como o de restaurantes.

O estudo teve como principais limitações: a escala de sete pontos, apesar de adequada, resultou em médias muito próximas, o que talvez pudesse ser amenizado com uma escala de 10 pontos. Também não houve a comprovação plena da validade estrutural do modelo inicialmente proposto, tendo em vista que algumas hipóteses não foram aceitas e o construto reclamação não foi confirmado no modelo para os serviços de restaurantes. As variávies de mensuração do construto lealdade apresentaram cargas fatoriais mais baixas que as médias gerais dos demais construtos, o que exige uma melhor avaliações desses indicadores utilizados no instrumento de coleta de dados, a partir de uma análise fatorial exploratória, apesar de não ter sido utilizada neste estudo, em razão do questionário ter sido construído com base em indicadores originais do instrumento ACSI. Generalizações dos resultados para outras cidades do país também não são possíveis, tendo em vista que a pesquisa se restringiu a uma única cidade do estado do Rio Grande do Sul. Pecularidades regionais e culturais podem influenciar nos resultados de outras regiões do país. 


\section{REFERÊNCIAS}

ACSI (2012). Global leadership. Apresenta informações gerais e relatórios da metodologia ACSI e CSI. Recuperado de http://www.theacsi.org

Akyol, A., Akehurst, G. (2003). An investigation of export performance variations related to corporate export market orientation. European Business Review, 15 (1), 5-19. doi: 10.1108/09555340310455155

Anderson, E. W., Fornell C., \& Mazvancheryl, S. K. (2004). Customer satisfaction and shareholder value. Journal of Marketing, 68, 172-185. doi: 10.1509/jmkg.68.4.172.42723

Anderson, E., Fornell, C., \& Lehmann, D. (1994). Customer satisfaction, market share and profitability: findings from Sweden. Journal of Marketing, 58, 53-66. doi: 10.2307/1252310

Andreassen, T., \& Lindestad, B. (1998). Customer loyalty and complex services: the impact of corporate image on quality, customer satisfaction and loyalty for customers with varying degrees of service expertise. International Journal of Service Industry Management, 9(1), 7-23. doi: 10.1108/09564239810199923

Angnes, D. L., \& Mello Moyano, C. A. (2013). Atributos de escolha em serviços de restaurantes: Um estudo exploratório. Revista Brasileira de Pesquisa em Turismo, 7(2), 317-336. doi: 10.7784/rbtur.v7i2.607

ANEEL (2012). Índice ANEEL de satisfação do consumidor. Recuperado de http://www.aneel.gov.br/arquivos/PDF/LIGHT_2010.pdf

Bateson, J. E. G., \& Hoffman, K. D. (2001). Marketing de serviços (4a ed.). Porto Alegre: Bookman.

Brei, V. A., \& Liberali Neto, G. (2006). O uso da técnica de modelagem de equações estruturais na área de marketing: um estudo comparativo entre publicações no Brasil e no exterior. Revista de Administração Contemporânea (RAC), 10(4), 131-151. doi: 10.1590/S1415-65552006000400007

Cardozo, R. N. (1965). An experimental study of customer effort, expectation, and satisfaction. Journal of Marketing Research, 2, 244-249. doi: 10.2307/3150182

Churchill, G., \& Suprenant, C. (1982). An investigation into the determinants of customer satisfaction. Journal of Marketing Research, 19, 491-504. doi: 10.2307/3151722

Flanagan, J. C. (1954). The critical incident technique. Psychological Bulletin, 51(4), 327-358. doi: $10.1037 / \mathrm{h} 0061470$

Fornell, C. (1992). A national customer satisfaction barometer: the Swedish experience. Journal of Marketing, 56, 6-21. doi: 10.2307/1252129 
Fornell, C. (2007). Satisfied customer: Winners and Losers in the Battle for Buyer Preference. New York: Palgrave Macmillan.

Fornell, C., Johnson, M. D., Anderson, E. W., Cha, J., \& Bryant B. E. (1996). The American Customer Satisfaction Index: nature, purpose and findings. Journal of Marketing, 60, 7-18. doi: 10.2307/1251898

Fornell, C., Mithas, S., Morgeson, F. V., \& Krishnan, M. S. (2006). Customer satisfaction and stock prices: high returns, low risk. Journal of Marketing, 70, 3-14. doi: 10.1509/jmkg.2006.70.1.3

Hair, J. F., Anderson, R. E., Tatham, r. L., Black, W. C., \& Babin, B. J. (2009). Análise multivariada de dados (6a ed.). Porto Alegre: Bookman.

Ladhari, R. (2009). A review of twenty years of SERVQUAL research. International Journal of Quality and Service Sciences, 1(2), 172-198. doi: 10.1108/17566690910971445

Lam, S. Y., Shankar, V., Erramilli, M. K., \& Murthy, B. (2004). Customer value, satisfaction, loyalty, and switching costs: an illustration from a business-to-business service context. Journal of the Academy of Marketing Science, 32(3), 293-311. doi: 10.1177/0092070304263330

Lockwood, A. (1994). Using service incidents to identify quality improvement points. International Journal of Contemporary Management, 6, 75-80. doi: 10.1108/09596119410052170

Lopes, H. E. G., Pereira, C. C. P., \& Vieira, A. F. S. (2009). Comparação entre os modelos norte-americano (ACSI) e europeu (ECSI) de satisfação do cliente: um estudo no setor de serviços. Revista Administração Mackenzie (RAM), 10(1), 161-187.

Malhotra, N. (2012). Pesquisa de marketing: uma orientação aplicada (6a ed.). Porto Alegre: Bookman.

Marchetti, R., \& Prado, P. H. M. (2004). Avaliação da satisfação do consumidor utilizando o método de equações estruturais: um modelo aplicado ao setor elétrico brasileiro. Revista de Administração Contemporânea (RAC), 8(4), 9-32. doi: 10.1590/\$1415-65552004000400002

Mello, C. H. P. (2010). Gestão da qualidade. São Paulo: Pearson.

Moura, A., \& Gonçalves, C. (2005). Modelo de satisfação ACSI modificado no setor de telefonia móvel. Revista de Administração de Empresas (RAE), 45, 72-85.

Mowen, J., \& Minor, M. (2003). Comportamento do consumidor. São Paulo: Prentice-Hall.

Oliver, R. L. (1980). A Cognitive model of the antecedents and consequences of satisfaction decisions. Journal of Marketing Research, 17, 460-469. doi: 10.2307/3150499 
Parasuraman, A., \& Grewal, D. (2000). The impact of technology on the quality-value-loyalty chain: a research agenda. Journal of the Academy of Marketing Science, (28), 168-174. doi: $10.1177 / 0092070300281015$

Santos, C. P., \& Fernandes, D. H. (2008). A recuperação de serviços como ferramenta de relacionamento e seu impacto na confiança e lealdade dos clientes. Revista de Administração de Empresas (RAE), 48(1), 1024.

Souki, G. Q., Madureira, K. T., Gonçalves Filho, C., \& Reis Neto, M. T. (2010). Desenvolvimento e validação de uma escala de atributos utilizados pelos consumidores na escolha de restaurantes de comida a quilo. Revista de Administração da Unimep, 8(2), 72-90.

Spreng, R., Mackenzie, S., \& Olshavsky, R. (1996). A reexamination of the determinants of consumer satisfaction. Journal of Marketing, 60, 15-32. doi: 10.2307/1251839

Tinoco, M. A. C., \& Ribeiro, J. L. D. (2008). Estudo qualitativo dos principais atributos que determinam a percepção de qualidade e de preço dos consumidores de restaurantes a la carte. Gestão da Produção, 15(1), 73-87. doi: 10.1590/S0104-530X2008000100008

Urdan, A., \& Rodrigues, A. (1999). O modelo do índice de satisfação do cliente norte-americano: um exame inicial no Brasil com equações estruturais. Revista de Administração Contemporânea (RAC), 3(3), 109-130. doi: 10.1590/S1415-65551999000300006

Woodruff, R. (1997). Customer value: the next source for competitive advantage. Journal of the Academy of Marketing Science, 25(2), 139-153. doi: 10.1007/BF02894350

Zeithaml, V. A. (1981). How Consumer Evaluation Processes Differ Between Goods and Services. In J. H. Donnelly \& W. R. George. Marketing of services. Chicago: American Marketing Association, pp. 186-90.

Zeithaml, V. A. (1988). Consumer perception of price, quality and value: a means-end model and synthesis of evidence. Journal of Marketing, 52. doi: 10.2307/1251446

Zeithaml, V. A., \& Bitner, M. J. (2003). Marketing de serviços: a empresa com foco no cliente (2a ed.). Porto Alegre: Bookman.

Zeithaml, V. A., Berry, L. L., \& Parasuraman, A. (1996). The behavioral consequences of service quality. Journal of Marketing, 60(2), 31-46. doi: 10.2307/1251929

Zineldin, M. (2006). The royalty of loyalty: CRM, quality and retention. Journal of Consumer Marketing, 23 (7), 430-437. doi: 10.1108/07363760610712975 